This item was submitted to Loughborough's Research Repository by the author.

Items in Figshare are protected by copyright, with all rights reserved, unless otherwise indicated.

\title{
Elastodynamic transient analysis of a four-cylinder valvetrain system with camshaft flexibility
}

PLEASE CITE THE PUBLISHED VERSION

PUBLISHER

Professional Engineering Publishing / @ IMECHE

VERSION

VoR (Version of Record)

LICENCE

CC BY-NC-ND 4.0

\section{REPOSITORY RECORD}

Teodorescu, M., M. Kushwaha, Homer Rahnejat, and D. Taraza. 2019. "Elastodynamic Transient Analysis of a Four-cylinder Valvetrain System with Camshaft Flexibility”. figshare. https://hdl.handle.net/2134/4800. 
This item was submitted to Loughborough's Institutional Repository (https://dspace.lboro.ac.uk/) by the author and is made available under the following Creative Commons Licence conditions.

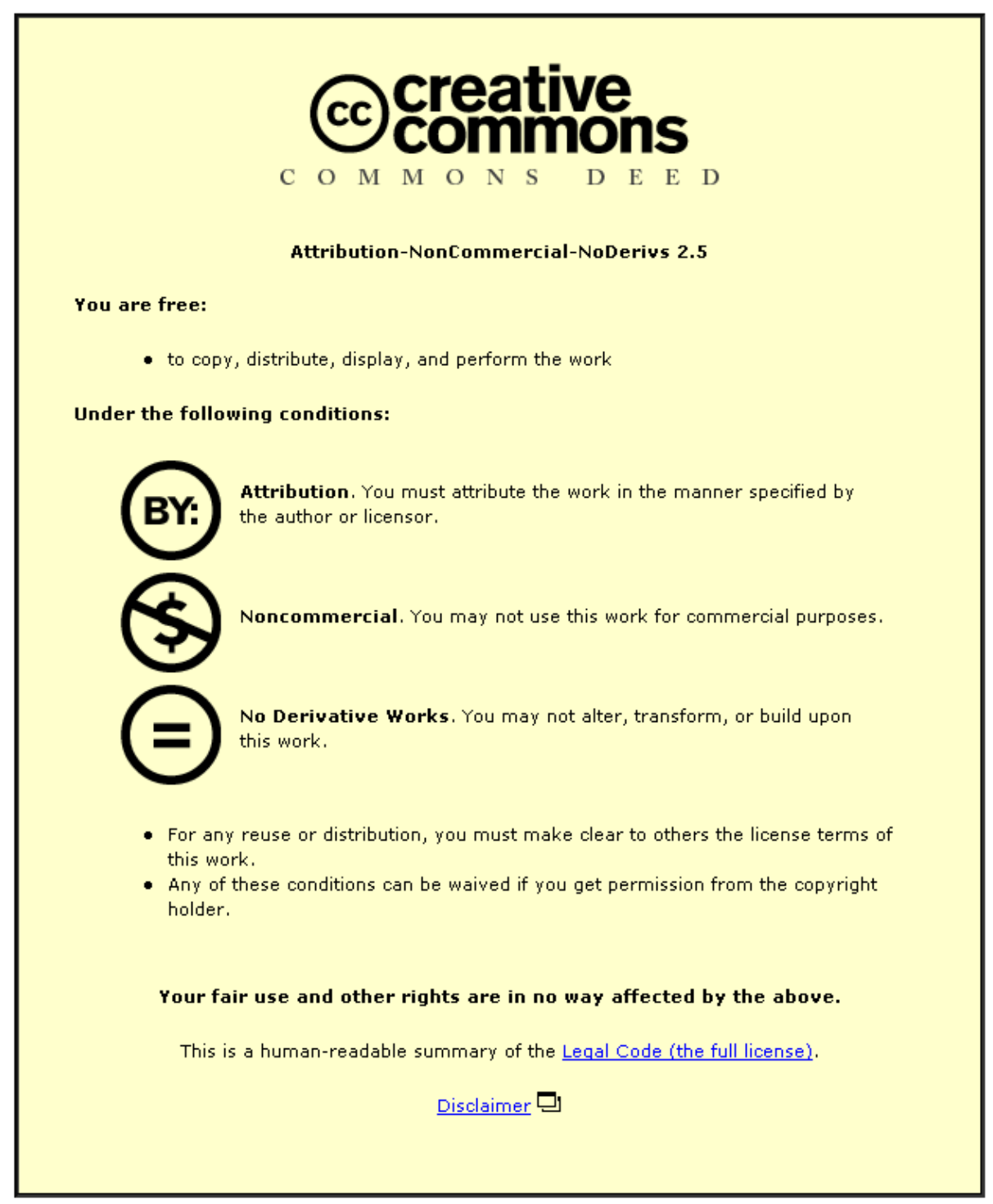

For the full text of this licence, please go to: http://creativecommons.org/licenses/by-nc-nd/2.5/ 


\title{
Elastodynamic transient analysis of a four-cylinder valvetrain system with camshaft flexibility
}

\author{
M Teodorescu ${ }^{1}$, M Kushwaha ${ }^{1}$, H Rahnejat ${ }^{1 *}$, and D Taraza ${ }^{2}$ \\ ${ }^{1}$ Wolfson School of Mechanical and Manufacturing Engineering, Loughborough University, Loughborough, UK \\ ${ }^{2}$ Department of Mechanical Engineering, Wayne State University, Michigan, USA
}

The manuscript was received on 2 August 2004 and was accepted after revision for publication on 27 October 2004.

DOI: 10.1243/146441905X9962

\begin{abstract}
This paper presents an analysis of a line of valvetrains in a four-cylinder, four-stroke in-line diesel engine. The method highlighted in this paper predicts the vibration signature together with the prevailing contact conditions and frictional characteristics exhibited in the valvetrain system. This integrated dynamic and tribological investigation provides a practical approach that can be used during the design or the evaluation phase of automotive valvetrain systems.
\end{abstract}

Keywords: multi-body dynamics, valvetrain system, bending vibrations, torsional vibrations, frictional contacts, cam-tappet friction

\section{INTRODUCTION}

There are a number of problems that arise during the design of valvetrain systems, including engine breathing requirements, mechanical and frictional losses, as well as noise and vibration concerns. A holistic approach, which ideally takes into account all these aspects and their interactions, and may be referred to as a 'multiphysics analysis', is sought to resolve this problem. In addition, there are many sources that give rise to the transient nature of the problem. These include the flotation of the point of contact between, for example, the cam and the flat follower surface, which gives rise to combined lubricant traction and boundary friction. This is due to the interactions of mating surfaces in the real physical system (see references [1] and [2]). Such considerations are often omitted in numerical analysis work. Excessive friction can lead to torsional vibration of the camshaft owing to its flexibility, a phenomenon commonly referred to as camshaft wind-up, just prior to cam nose-follower contact, and subsequent wind-down after cam nose-follower contact, as described by Koster [3] and Kushwaha et al. [4]. Another source of transience is the valve

\footnotetext{
* Corresponding author: Wolfson School of Mechanical and Manufacturing Engineering, University of Loughborough, Loughborough, Leicestershire LE11 3TU, UK.
}

spring surge, which occurs above certain camshaft speeds and can lead to two major concerns. Firstly, it can cause contact separation between the cam and the follower and subsequent rebound with impact-induced implications. These are usually noted in terms of momentary loss of lubrication owing to contact separation and subsequent impact forces that can yield high pressures, which may lead to the fatigue spalling problem. Secondly, the valvespring surge effect [4] invariably causes valve toss or flutter which has many associated problems, including wear and fatigue of the usually poorly lubricated valve-valve seat contact and small fluctuations in the combustion pressure.

The circumferential variation in the effective contact profile of the camfollower leads to a transient effect in the kinematics of the contact and corresponding variations in the lubricant film thickness, especially leading to inlet boundary reversals prior to and after the cam nose contact with momentary cessation of entraining motion. Although a small lubricant film thickness is maintained by the squeeze film effect and rapid replenishment occurs owing to short transit time (see references [5] and [6]), this phenomenon can lead to the aforementioned boundary or mixed lubrication conditions.

This paper outlines an initial multiphysics approach, consisting of constrained multi-body dynamics, component flexibility, and kinematics and frictional behaviour of contacts. There also exists a 
dearth of experimental as well as closed-form analytical verification of numerical models, which has been tackled to a certain extent in this paper.

\section{THEORETICAL FORMULATION}

The above-mentioned problems give an indication of the complexity of valvetrain analysis. Therefore, analysis methods should be developed carefully and devised in incremental steps in order to yield realistic outcomes.

The devised model is a line of valves for a fourcylinder in-line diesel engine (see Fig. 1). The present study firstly proposes a set of analytical mathematical modules, which simulate the tribodynamic behaviour of individual components of a standalone valvetrain (see Fig. 2), and secondly combines them to constitute the entire line of valvetrains through a flexible camshaft. Each component is characterized by its inertial properties and its compliance: stiffness and damping.

\subsection{Kinematics of cam-flat follower contact}

Figure 2 shows the flat follower contacting at a point $\mathrm{P}$ on the cam flank. The fixed global coordinate system $X Y$ is positioned at the centre of the cam, $O$. The angular velocity of the camshaft is $\omega$, as shown in the figure, with the valve lift indicated by $s$.

It is important to determine the speed of entraining motion of the lubricant in the contact, in order to obtain the film thickness, which, together with the contact deflection, accounts for the

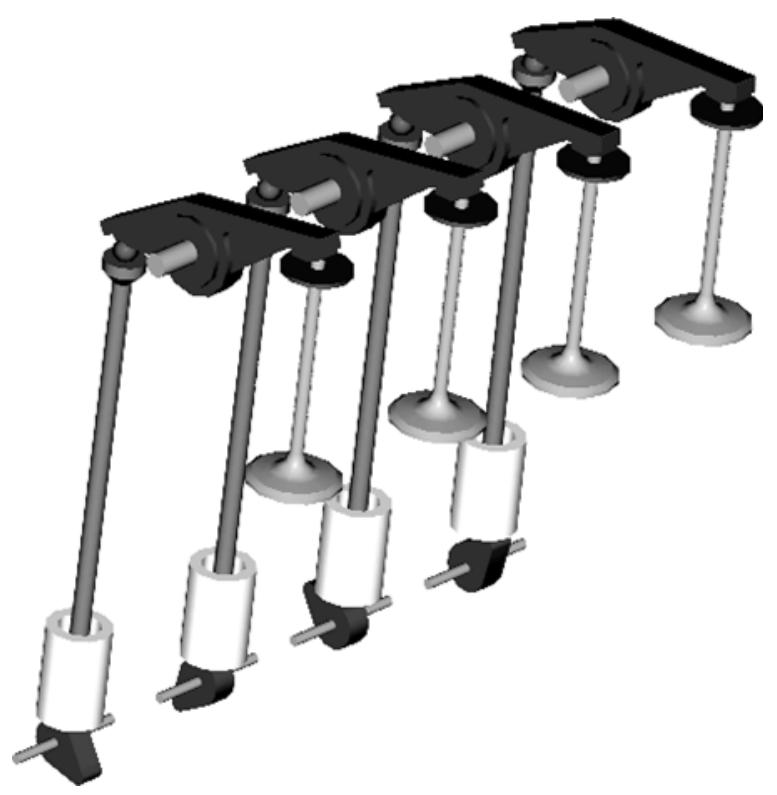

Fig. 1 Schematic representation of a valvetrain system for a four-cylinder in-line engine

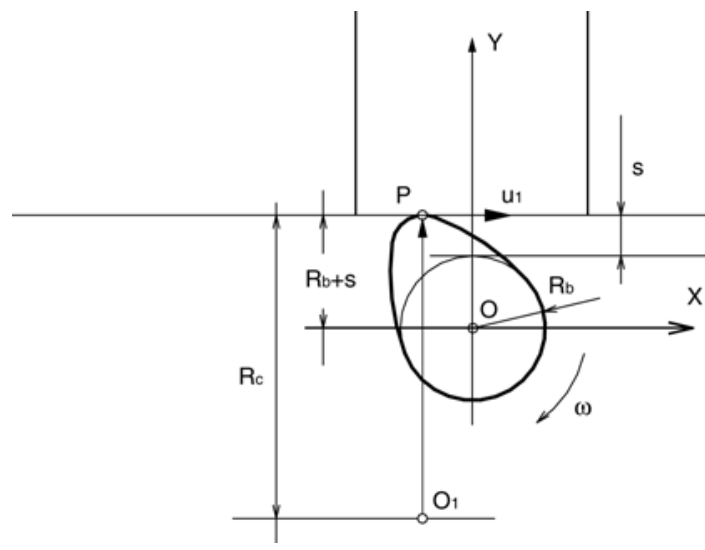

Fig. 2 Geometry of the cam-tappet contact

instantaneous gap formation between the loadbearing surfaces. The speed by which the lubricant is pulled into the contact by the rotation of the cam is given as

$$
u=\frac{u_{1}+u_{2}}{2}
$$

It should be noted that, for simplicity, the surface speed of the flat follower is considered to be zero, when neglecting the tappet spin. Therefore, the speed of lubricant entrainment is simply half that of the instantaneous speed of the cam surface at the contact region. This is clearly

$$
u_{1}=R_{\mathrm{c}} \omega
$$

Note that the instantaneous radius of curvature at the point of contact is also subjected to a geometrical acceleration, caused by the cam lift, which causes tappet acceleration in an upward direction. Hence

$$
R_{\mathrm{c}}=R_{\mathrm{b}}+s+\frac{\ddot{s}}{\omega^{2}}
$$

Substituting $R_{\mathrm{c}}$ from equation (3) into equation (2), and subsequently $u_{1}$ into equation (1), the speed of entraining motion is obtained as

$$
u=\left[R_{\mathrm{c}}+\frac{\ddot{s}}{\omega^{2}}\right] \frac{\omega}{2}
$$

\subsection{Tribological conditions in the concentrated contact}

The lubricated contact condition between the cam and the flat follower is very complex indeed. The regime of lubrication alters through the cam cycle owing to significant changes in the contact force and the velocity of lubricant entrainment. 
In the most loaded parts of the cycle, on the cam nose and in the vicinity of it, particularly at low valve accelerations, the regime of lubrication is piezoviscous elastic (EHL). Where the inlet reversals take place with cessation of entraining motion, a mixed regime of lubrication may result, and in the extreme cases boundary lubrication may act alone. On the flanks of the cam, conditions are likely to be less severe, with a predominant hydrodynamic regime of lubrication. Wherever the entraining velocity is high, tractive forces may play an important role owing to large lubricant shearing rates that may lead to non-Newtonian lubricant behaviour. When the lubricant thickness is less than 3 times the root mean square of the roughness or the composite surfaces, the ensuing conditions can promote significant friction owing to asperity interactions with or without lubricant tractive contribution. Therefore, it is clear that a practical contact model should comprise contact load generation through lubricant pressures as well as friction due to combined boundary and fluid film contributions.

\subsection{Lubricant reaction}

Ideally, the transient lubricated contact condition would be investigated through simultaneous solution of Reynolds equation with the inclusion of the elastic squeeze-film effect and the elastic film shape for this finite line concentrated contact conjunction. Solutions of this type have been reported recently by Kushwaha and Rahnejat [6], but with time-intensive computation of the order of hundreds of hours in CPU time. Clearly, from a practical viewpoint, a realistic solution should be of the order of a few CPU seconds per time step of integration. Thus, a simplified yet sensible and representative method is required. Use is made of extrapolated oil-film formulae for the right contact geometry, in this case for a finite line configuration, in which the width of the cam accounts for the finite length of the contact, and a typical geometry is best described by a 'dog-bone' or a 'dumb-bell' shape. Equations for such conjunctions have been obtained by Mostofi and Gohar [7] and Rahnejat [8], who have employed the same for the study of lubricated gear teeth meshing dynamics [9] and found very good agreement with the experimental findings of Dareing and Johnson [10].

The extrapolation equation for the central oil-film thickness for such conjunctions was derived for combined entraining and squeeze-film actions by Rahnejat [8] and is given as

$$
h_{0}^{*}=1.67 G^{* 0.421} U^{* 0.541} W^{* 0.059} \mathrm{e}^{-96.775 w_{\mathrm{s}}^{*}}
$$

The dimensionless parameters on the right-hand side of the above equation relate to material combinations, $G^{*}$, rolling viscosity, $U^{*}$, and loading conditions, $W^{*}$.

The squeeze-roll ratio is given by the exponent

$$
w_{\mathrm{s}}^{*}=\frac{1}{u_{\mathrm{e}}} \frac{\mathrm{d} h_{0}}{\mathrm{~d} t}=\frac{1}{u_{\mathrm{e}}} \frac{\mathrm{d} h_{0}}{\mathrm{~d} X} \frac{\mathrm{d} X}{\mathrm{~d} t}=\frac{1}{u_{\mathrm{e}}} u_{\mathrm{e}} \frac{\mathrm{d} h_{0}}{\mathrm{~d} X}=\frac{\mathrm{d} h_{0}}{\mathrm{~d} X}
$$

This clearly shows that the squeeze-roll ratio is simply the slope of the film with respect to the direction of entraining motion (i.e. the slope in the positive $X$ direction in Fig. 2). Larger negative slopes indicate a more pronounced wedge shape, encouraging lubricant film entrapment, while a zero slope corresponds to a flat-type film, and a positive slope indicates reduction in the wedge effect. Referring back to equations (6) and (5), the negative values for $\mathrm{d} h_{0} / \mathrm{d} t$ increase the film thickness. This corroborates the above argument and leads to an increased load-carrying capacity. It should be noted that the squeeze-roll ratio or the slope of the film is always a very small number.

The contact load is the integrated contact pressure distribution, which is the same as the force responsible for the local deformation of the contact. The contact deflection can be approximated to

$$
\delta_{0}=\frac{W}{k}
$$

\subsection{Contact friction}

The contact friction as described above is assumed to be the result of a combination of boundary friction and viscous traction. The surface roughness, $R_{\mathrm{a}}$, of the mating members is $0.1 \mu \mathrm{m}$, making their composite r.m.s. value approximately $\sigma=0.15 \mu \mathrm{m}$. Boundary friction contributes to the mechanism of friction for $h_{0} / \sigma<3$. This friction force is determined by the relationship proposed by Greenwood and Tripp [11] and is given as

$$
F_{\mathrm{b}}=\tau_{0} A_{\mathrm{a}}+m W_{\mathrm{a}}
$$

The first term on the right-hand side of equation (8) represents the non-Newtonian shear force caused by the small amount of low shear strength surface film adhering to the interacting asperity peaks. The load carried by the asperities is also stated by Greenwood and Tripp [11] as

$$
W_{\mathrm{a}}=\frac{8 \sqrt{2}}{15} \pi(\zeta \beta \sigma)^{2} \sqrt{\frac{\sigma}{\beta}} E_{\mathrm{r}} A F_{5 / 2}(\lambda)
$$


The area occupied by the asperity peaks is given by

$$
A_{\mathrm{a}}=\pi^{2}(\zeta \beta \sigma)^{2} A F_{2}(\lambda)
$$

The viscous friction is given (see references [11] and [12]) as

$$
F_{\mathrm{v}}=\tau\left(A-A_{\mathrm{a}}\right)
$$

where the non-Newtonian shear stress is given as a function of normal load components (see reference [13]) as

$$
\tau=\tau_{0}+\gamma \bar{W}
$$

where

$$
\bar{W}=\frac{W-W_{\mathrm{a}}}{A-A_{\mathrm{a}}}
$$

Thus

$$
F_{\mathrm{v}}=\tau_{0}\left(A-A_{\mathrm{a}}\right)+\gamma\left(W-W_{\mathrm{a}}\right)
$$

Now the total friction force becomes

$$
F_{\mathrm{f}}=F_{\mathrm{b}}+F_{\mathrm{v}}=\tau_{0} A+\gamma W+(m-\gamma) W_{\mathrm{a}}
$$

For more information, readers are referred to work by Teodorescu et al. [12] and Teodorescu [1].

\subsection{Dynamics of a single valvetrain mechanism}

The arrangement of a single valvetrain mechanism can be modelled as a multi-body system, comprising a number of mass/inertial elements connected to each other by a series of restraining elements (see Fig. 3). These elements represent the sources of compliance in the system, such as the valve spring stiffness and contact compliance between the cam and the flat follower. To determine the translational equivalent mass corresponding to the rotational rocker arm, the current research considers a kinetic energy approach which is explained in Appendix 2. The two inertial elements in each module of the line of valves represent the mass of the valve and all its attachments (including the retainer and one-third of the non-negligible mass of the valve spring), that of the pushrod/flat follower, and the proportion of the rocker arm in translation. The various stiffness and damping components in the model are described in the notation. The 'floor' movement in this model, denoted by $s$, represents the movement of the surface of the cam, which adheres to the flat follower surface by the contact compliance indicated by $k_{1}, c_{1}$.

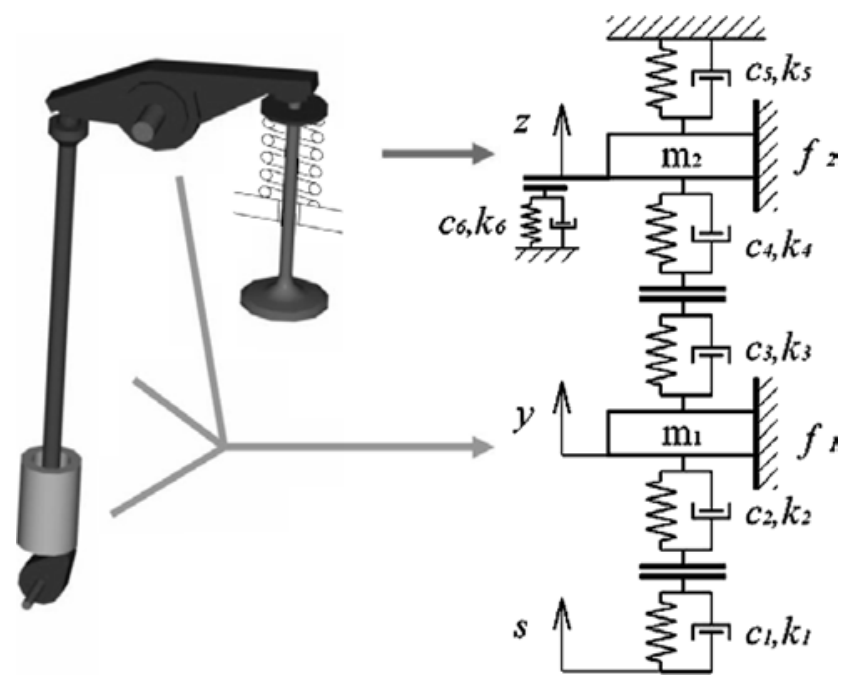

Fig. 3 Dynamic equivalent model for a single valvetrain system

The motion of the follower/pushrod assembly is denoted by $y$, whereas the movement of the valve itself is denoted by $z$. Therefore, the multi-body model is a two-degree-of-freedom system. The contacts at the load-bearing surfaces are depicted in the model in Fig. 3 by gaps shown with parallel horizontal lines. These gaps represent lubricated conjunctions, where loss of contact load can result in the separation effect referred to in the introduction section. In this paper only the lubricated conjunction between the cam and the flat follower is taken into account. The equivalent stiffness and the corresponding damping coefficient for this contact conjunction, as well as the compliance of structural elements of the system, such as the flexibility of the pushrod, the valve stem, and the rocker arm, are also taken into account. These are obtained by simple serial additive functions.

The two equations of motion for the single valvetrain model can be written in Newton-Euler formulation. For a generic mass $m_{i}$, which has a displacement $l_{\mathrm{i}}$, experiencing an applied force $F_{i}^{m}$, the equation of motion can be written as

$$
\begin{aligned}
& m_{i} \ddot{l}_{i}+c_{\mathrm{e}_{i}}\left(\dot{l}_{i}-\dot{l}_{i-1}\right)+k_{\mathrm{e}_{i}}\left(l_{i}-l_{i-1}\right)+c_{\mathrm{g}_{i}} \dot{l}_{i} \\
& \quad-k_{\mathrm{e}_{i+1}}\left(l_{i+1}-l_{i}\right)-c_{\mathrm{e}_{i+1}}\left(\dot{l}_{i+1}-\dot{l}_{i}\right)+F_{i}^{m}=0
\end{aligned}
$$

where

$$
k_{\mathrm{e}_{i}}=\frac{k_{i} k_{i+1}}{k_{i}+k_{i+1}}, \quad c_{\mathrm{e}_{i}}=\frac{c_{i} c_{i+1}}{c_{i}+c_{i+1}}, \quad i=1,2
$$

Clearly, three such equations of motion exist for this valvetrain model, which require simultaneous 
solution by a time-marching integration method. The method makes use of integration, based on backward difference formulae with first-order approximation in the case of first derivatives, and a trapezoidal rule for the second derivatives with respect to time

$$
\dot{l}_{i}=\frac{l_{i}-l_{i-1}}{\Delta t}, \quad \ddot{l}_{i}=\frac{l_{i+1}-2 l_{i}+l_{i-l}}{\Delta t}
$$

The model was built in a modular fashion, and hence it can be easily adapted for different valvetrain configurations. If the desired configuration does not include a pushrod, its representative mass is removed from the equivalent mass, $m_{\mathrm{l}}$, and the rest of the simulation remains unaffected.

\subsection{Camshaft flexibility}

The dynamic coupling between the separate valvetrains is accounted for through the inclusion of an elastic camshaft, in which both the bending and torsional vibrations are considered. The ideal solution requires a comprehensive analysis of a tribodynamic model which provides simultaneous solution for each individual mass of the valvetrain, for both bending and torsional modes of vibration of the camshaft, as well as the lubricated contact between the contiguous surfaces. Such an approach is quite accurate but requires an excessive amount of computation time and storage memory owing to the very large number of degrees of freedom. Consequently, such a method is attractive from a mathematical point of view but less effective from a practical perspective within industrial time-scales.

In the current research, each individual valvetrain was simulated using the dynamic model previously validated for a single-cylinder engine. However, for the simplified case of a single-cylinder engine, the cam angular velocity was considered constant, neglecting the camshaft possible bending and torsional vibrations, and consequently the excitation (the valve lift, $x$, in Fig. 3) was a function of time only. For the extended case of a multicylinder engine, the camshaft dynamics cannot be neglected anymore. The angular velocity of the camgear was considered to be constant and the excitation for each valvetrain was computed, considering the angular position of the camshaft as well as the bending and the torsional vibrations propagated through it to each individual cam. The approximate method chosen considers separate models for the torsional and bending vibrations, and the dynamic coupling between the two is ensured by the marching scheme used to solve the equations of motion.

Both camshaft models consider a number of inertial elements (concentrated masses for the bending vibrations and moments of inertia for the torsional oscillations) connected by restraining elements with stiffness and damping. The model has been developed in a generic manner, considering identical camshaft bearings between valvetrains and realistically stiffer bearings at each end of the camshaft. If the physical camshaft does not have one of the specified bearings, the model could be easily adapted by considering the corresponding stiffness equal to zero. Figure 4 shows a brief description of the model for bending vibrations and for torsional vibrations. The bending vibration model considers the oscillatory behaviour in the vertical plane only, neglecting the much smaller amplitude bending vibrations in the horizontal lateral plane.

For the numerical approach at each time step, the equation of motion for an inertial element is written similarly to equation (16), taking into account that, for the case of torsional vibrations, the concentrated force should be substituted by a concentrated momentum. The differential equations of motion are solved using backward difference formulae identical to those in equation (17).

The set of differential equations is solved by a marching scheme. At each time step, the dynamic

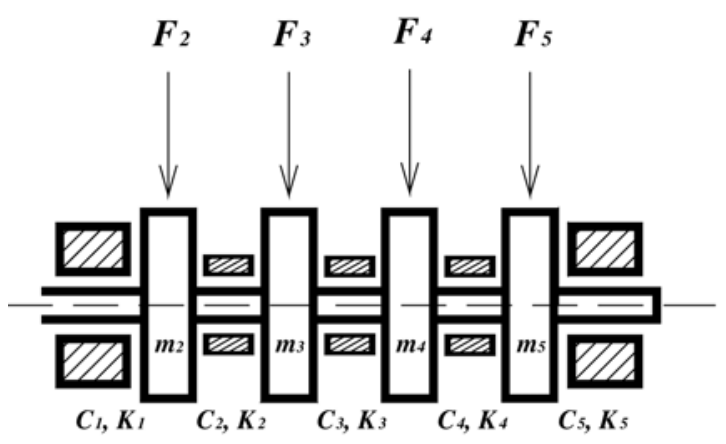

(a)

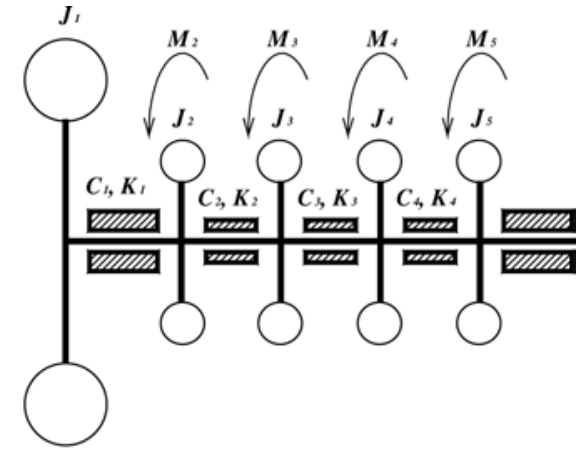

(b)

Fig. 4 Elastic models for (a) camshaft bending vibrations and (b) camshaft torsional vibrations 
response of the camshaft is computed on the basis of the vertical force and torque around the centre of the cam, predicted by each individual valvetrain model from the previous time step. Furthermore, within each time step, the camshaft dynamic model predictions are fed back into the individual valvetrain models to determine the force and the torque applied on each cam-tappet contact.

The main advantage of such a procedure over the more elaborate methods of solving for a line of valvetrains is the very fast and efficient solution, and very low memory requirements for the desired accuracy. Since the main purpose of this research is to demonstrate the prediction capabilities of an integrated method that accounts not only for the system dynamics but also for a larger range of physical phenomena, it is crucial firstly to develop a dynamic 'backbone' on which separate modules could be easily developed.

\section{EXPERIMENTAL VALIDATION FOR A SINGLE VALVETRAIN MECHANISM}

One of the major problems in the modelling work is validating the theoretical models with experimental data. The valvetrain simulation is built for a generic line of valvetrains. However, to validate the valvetrain dynamic model with experimental results, in the present study the dynamic model for an individual valvetrain system is experimentally validated for a single-cylinder diesel engine.

The experimental engine is a Deutz F1L 210D engine and is equipped with a pushrod-type valvetrain system (see Fig. 5).

The valve acceleration is experimentally measured using a piezoaccelerometer, mounted on the exhaust valve seat retainer. The location of the accelerometer

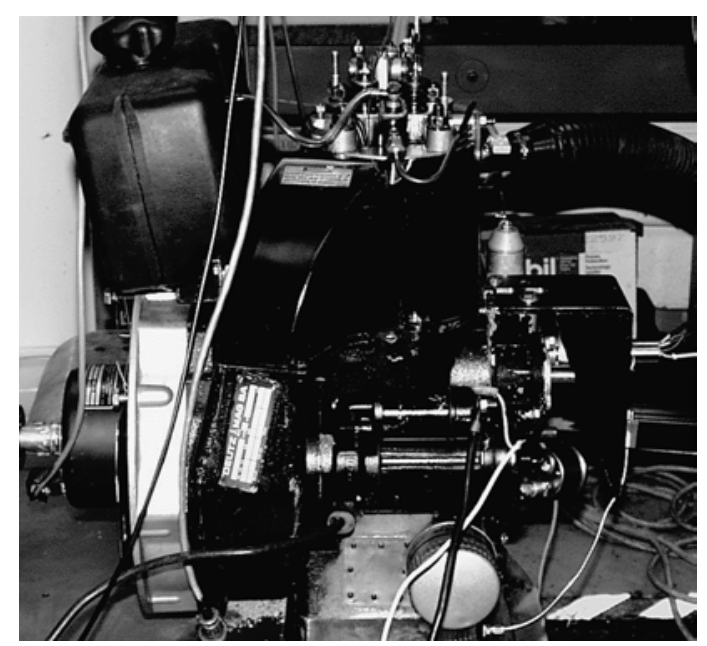

Fig. 5 Experimental engine (Deutz F1L 210D) is shown in Fig. 6. Based on the valvetrain geometry and on the measured valve acceleration, the actual motion of the cam is computed.

Since the experimental set-up is mounted on a running engine under normal operating conditions, the final result (especially at high engine speed) is heavily distorted owing to the engine noise. Consequently, the experimentally measured acceleration has to be carefully filtered before it is integrated twice to compute the valve lift. The numerical approach, together with a detailed description of the experimental set-up, has been reported by Teodorescu et al. [14]. To eliminate further the influence of external noise on the dynamic behaviour of the multicylinder valvetrain system, in the current research a high-order automotive-type polynomial valve has been fitted to the experimental valve lift. To choose the appropriate polynomial for the given problem, the approach described by Chen [15] was used as a starting point, and carefully adapted. The valve lift is considered to be symmetrical, with both events (opening and closing), and fully described by a polynomial function of the form

$$
\begin{aligned}
& x=x_{0}\left(1+\sum_{i}^{k} C_{i} \cdot \alpha^{r_{i}}\right), \\
& C_{i}=\frac{\Pi_{j=l ; j \neq i}^{k} r_{j}}{\Pi_{j=1 ; j \neq i}^{k}\left(r_{j}-r_{i}\right)}
\end{aligned}
$$

A large number of possible polynomials have been successively tested, and the closest agreement between the proposed valve lift and the experimentally measured one was reached for the following conditions

$$
\begin{gathered}
k=5, \quad r_{1}=2, \quad r_{2}=8, \quad r_{3}=14 \\
r_{4}=20, \quad r_{s}=26
\end{gathered}
$$

For the valve-train configuration shown in Fig. 3, the most flexible component (besides the valve spring) is the pushrod, which is responsible for the major vibration present in the harmonic spectrum of the measured valve acceleration. Figure 7 shows the comparison between the measured and simulated valve acceleration at $1735 \mathrm{r} / \mathrm{min}$.

The experimental engine is equipped with only one camshaft for both the intake and the exhaust valvetrains. As a result, the shock wave due to the cam-tappet impact on the intake side, at the beginning of the opening event, propagates to the exhaust side valvetrain as well, and is pointed out by an arrow in Fig. 7. However, since at this stage the model considers only the exhaust valvetrain, this phenomenon is not observed in the simulated valve lift acceleration. The very high-frequency vibration measured 


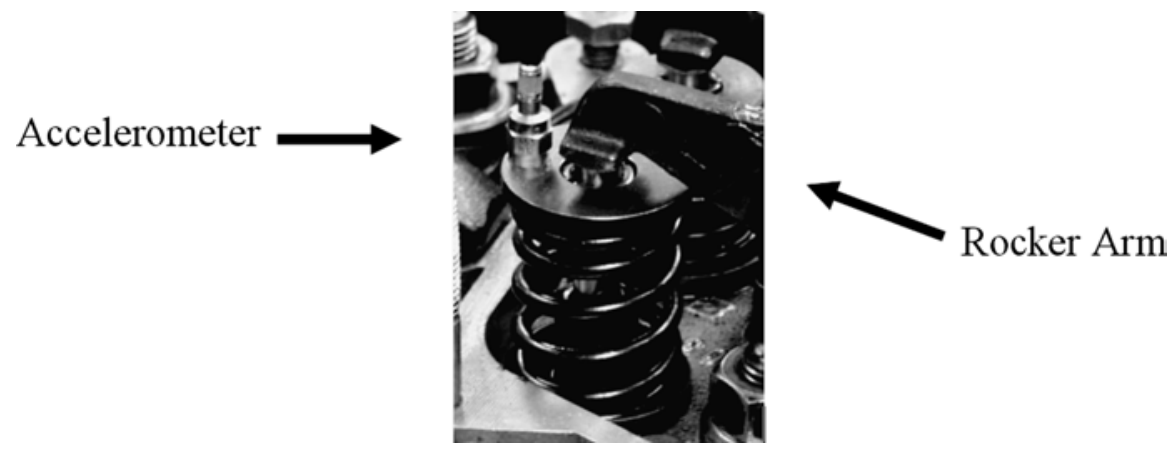

Fig. 6 Experimental set-up

at the end of the closing event is due to the impact between the valve and the valve seat. This behaviour is captured to a certain degree by the existing dynamic model. However, in order accurately to describe the very high forces generated by this impact, a separate impact model should be used. Owing to the complexity of integrating an, analytical model for the valve-valve seat impact into the existing valvetrain dynamic model, this aspect is left for further research and development at a later stage.

For a further validation of the dynamic model versus the experimental measurements, the harmonic spectrum of the predicted valve acceleration is compared with the spectrum of the measured one. The results are shown in Fig. 8 for the relevant section of the spectrum. The theoretical model predicts similar behaviour tote experimental observations, and the differences are mainly due to the simplifications needed to determine the two-mass model.

\section{RESULTS AND DISCUSSION}

Referring back to Fig. 1, a simulation study is undertaken in respect of a line of inlet valves of a

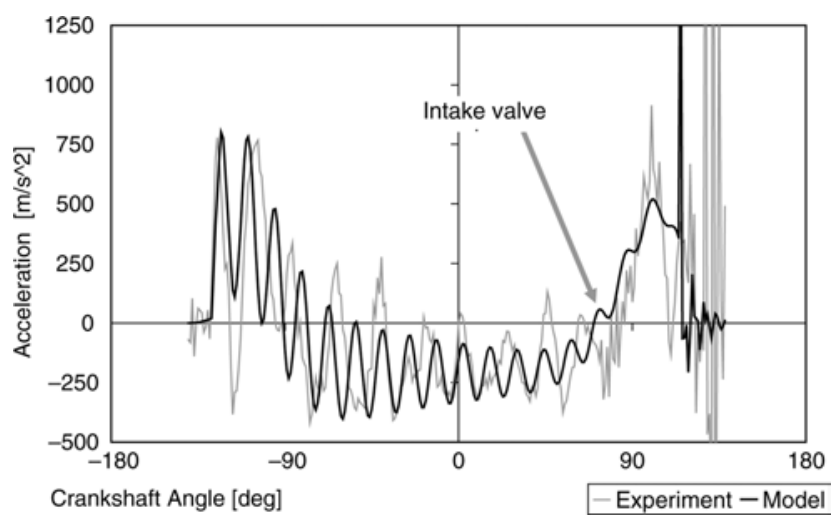

Fig. 7 Model predictions versus the experimental valve acceleration for an engine speed of $1735 \mathrm{r} / \mathrm{min}$ four-cylinder, four-stroke diesel engine with an engine cylinder firing order 1-3-4-2. The simulation was carried out on a dual-processor $2.66 \mathrm{GHz}$ (each) Pentium IV PC. The simulation time was typically less then $10 \mathrm{CPU}$ seconds. The analysis is undertaken for an engine speed of $1400 \mathrm{r} / \mathrm{min}$ ( $700 \mathrm{r} / \mathrm{min}$ on the camshaft), which renders low lubricant entrainment speed, yielding some of the worst conditions in tribological terms.

The polynomial automotive cam used in this analysis has a dwell-rise-return-dwell characteristic. Figure 9 shows the prevailing kinematic conditions for this type of cam throughout its cyclic action. The figure shows a rapid rise in tappet acceleration during the rise part of the cam cycle. This is followed by reduced acceleration at the cam nose-tappet contact, prior to the return part of the cycle. The return part of the cycle commences here in a symmetrical manner, as previously described.

Figure 10 shows the contact load between the cam-tappet pair for this line of valves according to the prescribed engine firing order. At this stage, and as a first approximation, the camshaft is considered infinitely rigid. One inlet valve cycle of all valves in the four-stroke process corresponds to

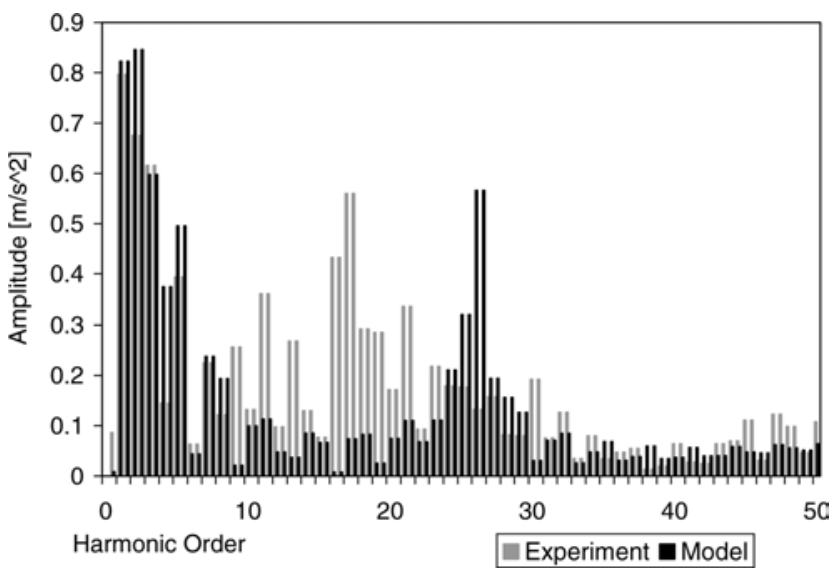

Fig. 8 Harmonic spectra for an engine speed of $1735 \mathrm{r} / \mathrm{min}$ 


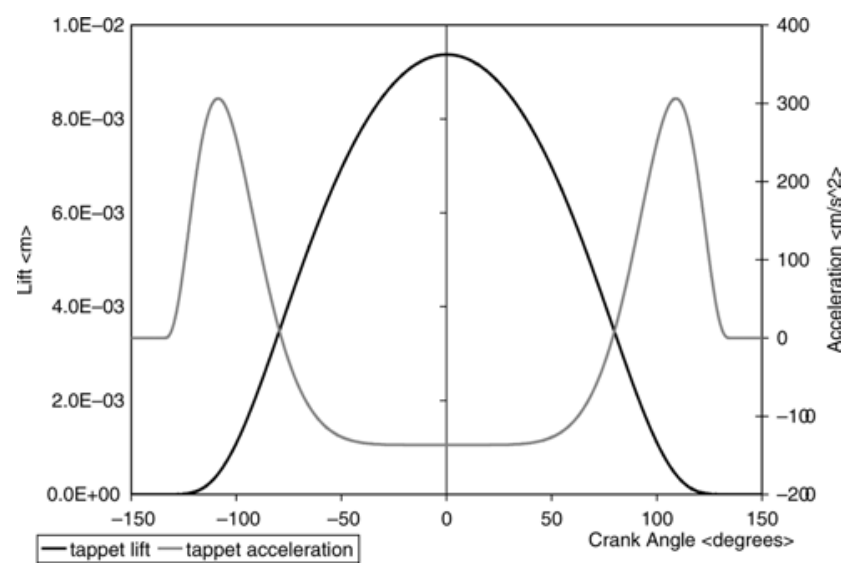

Fig. 9 Kinematic characteristics of the contact

two revolutions of the crankshaft. The repetitive shape indicates identical cycles per contact in each cycle, commencing with valve opening with a sharp rise in the contact load owing to geometrical acceleration caused by the cam lift. This is followed by the nose action, indicated by a semi-parabolic variation and a subsequent reduction in the contact load. The nose event is followed by an increase in the contact load owing to an increase in the reverse acceleration in the closing event. In each cycle of cam action, and on the cam rise, oscillatory behaviour in the contact load variation is observed owing to the 'wind-up' phenomenon on the camshaft, which is as a result of component flexibility in the valvetrain (previously described). The same effect is often noted to a much lesser extent on the return cycle, referred to as 'wind-down', because of the reduced deflection/ deformation in the compliant members. In practice, however, additional rapid oscillations are often observed at the instant of valve closure owing to loss of contact and subsequent valve bounce. This effect is not observed here, because the non-linear behaviour of the valve spring is not taken into account. This effect is known as valve flutter, caused by a surge effect in valve spring motion.

Validation of multi-body models is essential if the outcome of their predictions is to be relied upon. This can be carried out by comparison of numerical results with analytical solutions, which are fortunately available for valvetrain systems in the form of spectral composition of the vibration response of multivalve systems, namely the camstar. This is possible in the case of a line of valves acting on a rigid camshaft as in the case of the analysis performed here. Figure 11 shows the result of camstar analysis for this particular engine. For details regarding construction of a camstar, see work by Rahnejat [16].

For identical valve actions, and taking into account the firing phase vector, the camstar is constructed as a vector diagram with unit vectors representing the action of each valve in directions indicated by the function $i \gamma$, where $\gamma$ is the phase vector and, for the firing order $1-3-4-2$, is given by the vector $\{0, \pi / 2,3 \pi / 2, \pi\}^{\mathrm{T}}$. Now the value of $i$ can assume any integer number, representing a camshaft order, this being a multiple of the camshaft speed, or in other words, a half-multiple of the crankshaft speed. The value of $i \gamma$ determines the direction of the unit vector in a clockwise direction as measured by an angle subtended to the vertical. The vector sum of the resulting valve actions determines the contribution at that camshaft order. Figure 11 shows that all the contributions diminish, except for the fourth camshaft order and its multiples (i.e. the second engine order and its multiples). This fact can be used to verify the output of the numerical analysis. To do so, the instantaneous load on the rigid camshaft can be obtained by the sum of the actions of all the valves (i.e. the sum of contact forces) at any instant of time. This is shown in Fig. 12. The spectral composition of this applied force is obtained by fast

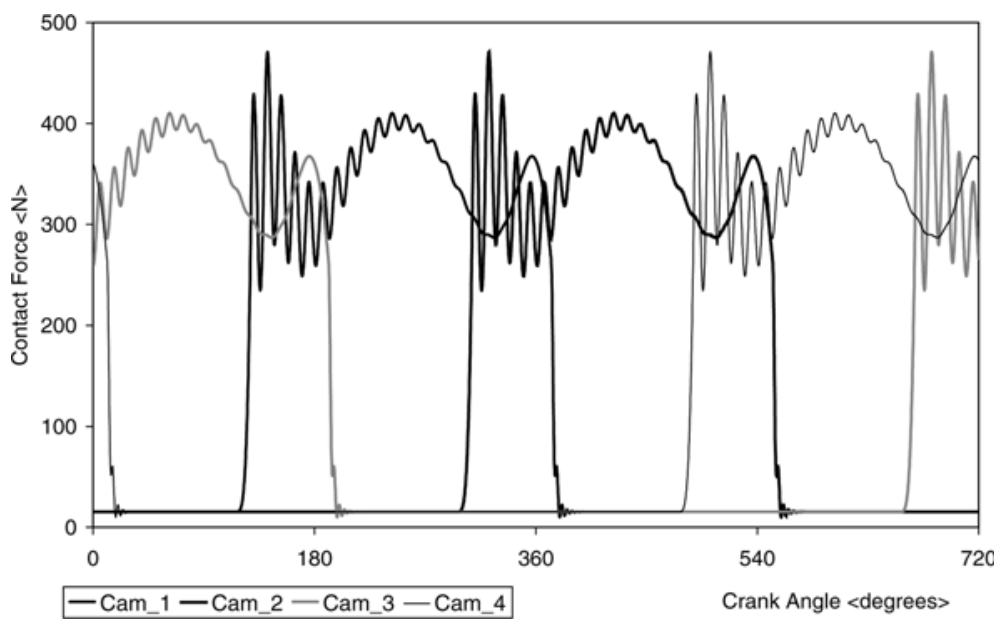

Fig. 10 Cam-tappet load for all four valvetrain mechanisms at $1400 \mathrm{r} / \mathrm{min}$ 


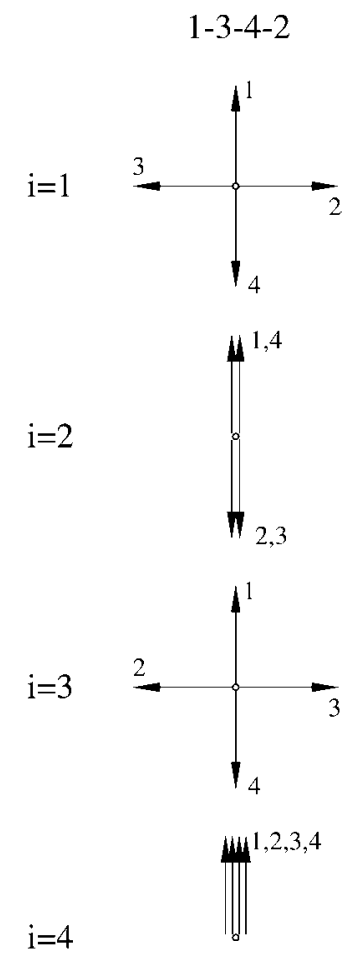

Fig. 11 Cam star for the 1-3-4-2 firing order

Fourier transform, (see Fig. 13), which shows agreement with the camstar. Note that the agreement is based on the frequency composition and not the amplitude, since simple camstar analysis, based on unit vector compositions, does not yield an amplitude, and nor does an FFT analysis (being essentially an averaging process) result in reliable amplitudes.

The frictional behaviour of the contact is responsible for the transmission of power and at the same time is critical to the safe operation of the valvetrain system. In a practical valvetrain analysis, the prediction of the lubricant film thickness responsible for the reduction of friction and improved engine

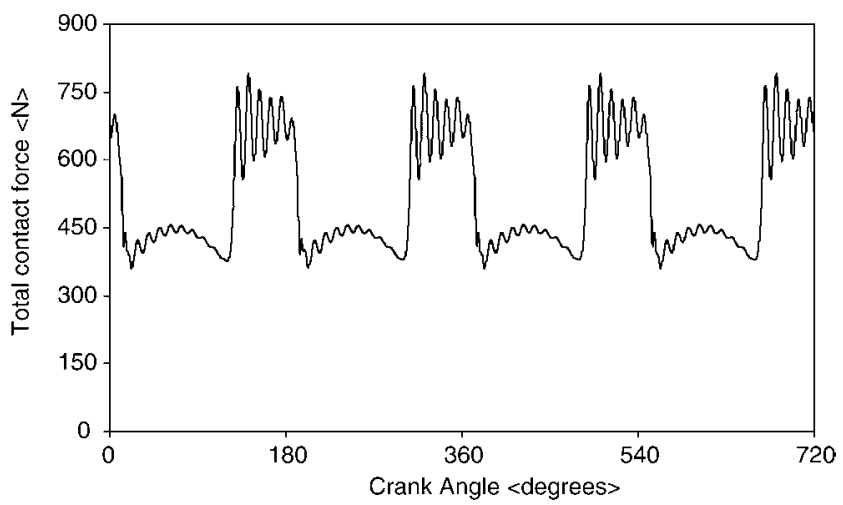

Fig. 12 Total load for the entire camshaft at $1400 \mathrm{r} / \mathrm{min}$

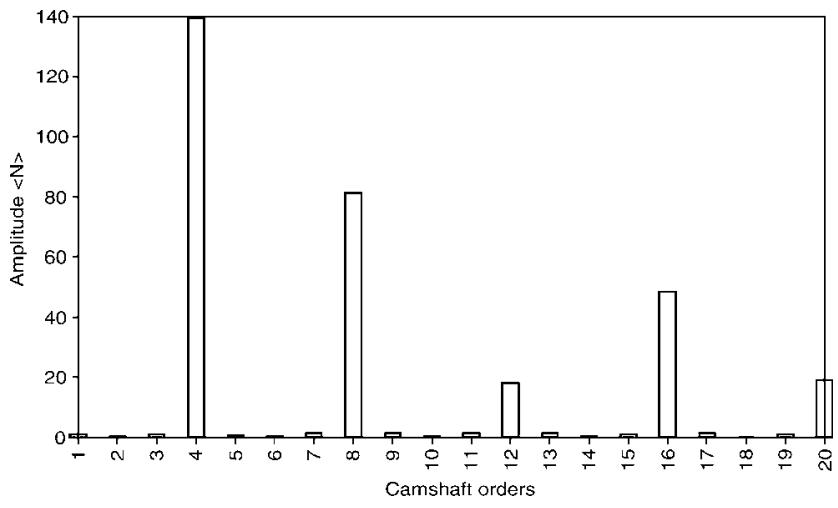

Fig. 13 Harmonic decomposition of the total contact force

efficiency is of interest. Figure 14 shows the central oil-Film thickness in the cam-flat follower contact. In line with the prevailing kinematic conditions, the lubricant film rises from a thickness of $0.1 \mu \mathrm{m}$ on the dwell part of the cycle to approximately $0.75 \mu \mathrm{m}$ on the flank regions of the cam action. It can be observed that the lubricant film falls rapidly under decelerative motion of the cam towards the cam nose owing to the decreasing speed of entraining motion, reaching a minimum film thickness prior to and in the vicinity of the cam nose, in this case at about $\pm 60^{\circ}$ to the cam nose position.

These positions of the oil-film thickness minima are due to inlet reversals, these being the positions that the entraining velocity changes direction and the lubricant flows in the opposite sense. With no squeeze-film action, the minimum film thickness would yield a value of zero. However, in this case, a very small film thickness exists because of lubricant entrapment by the squeeze-film caving action (although this appears to be zero in Fig. 14). This has been reported in a detailed lubrication analysis

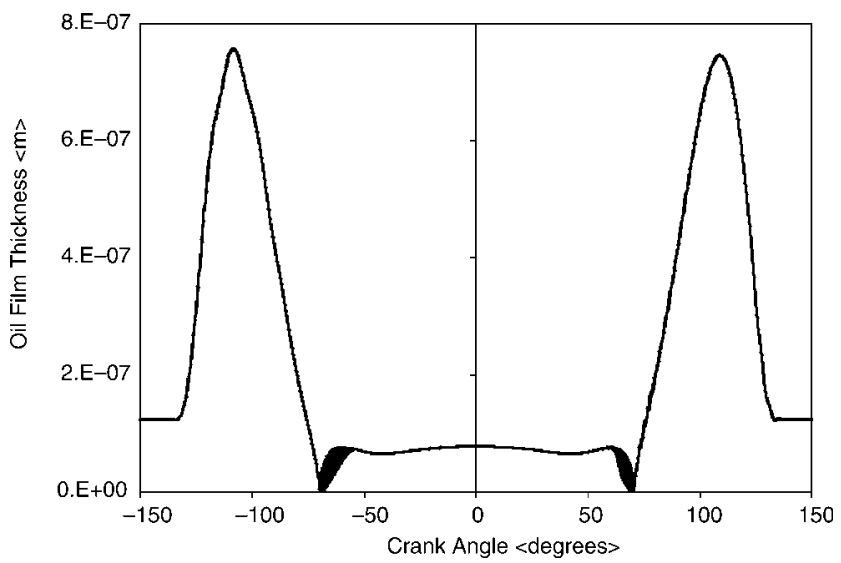

Fig. 14 Oil film thickness at $1400 \mathrm{r} / \mathrm{min}$ 


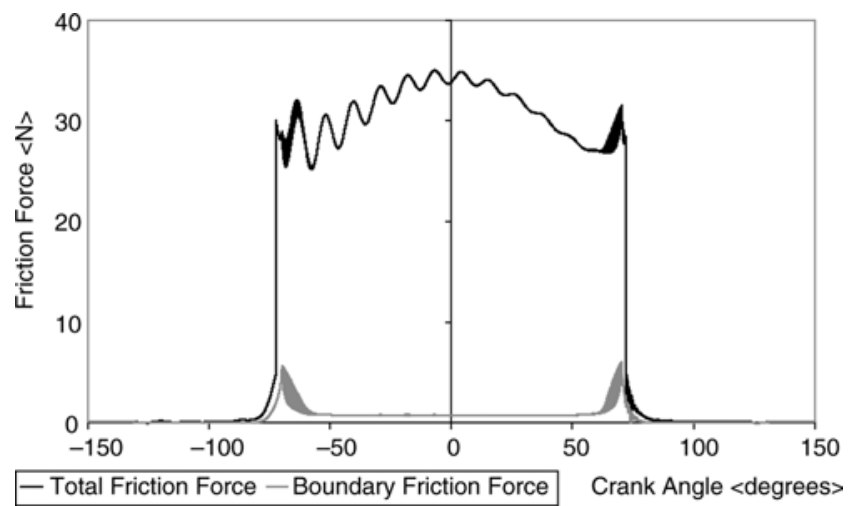

Fig. 15 Total friction force and the boundary friction force at $1400 \mathrm{r} / \mathrm{min}$

by Kushwaha et al. [17]. Referring back to section 2.4, it is clear that a mixed regime of lubrication would prevail in these regions, and in fact in a considerable part of the cam cycle. To ascertain the contribution due to boundary friction is an important undertaking, because any sizeable contribution would indicate enhanced friction and wear. Figure 15 shows the total friction force throughout the cam cycle with the corresponding contribution from the boundary friction force. It can be noted that the percentage share due to asperity interactions is less then 10 per cent even at the positions of the film thickness minima.

Figure 16 shows the predicted acceleration for all the four valvetrains at $2000 \mathrm{r} / \mathrm{min}$. By introducing camshaft flexibility into the theoretical model, individual valvetrains are dynamically coupled, and the excitation fed back into each of the valvetrain dynamic models is influenced remotely by the behaviour of the others. The very high acceleration peaks at the end of each of the closing events has a much

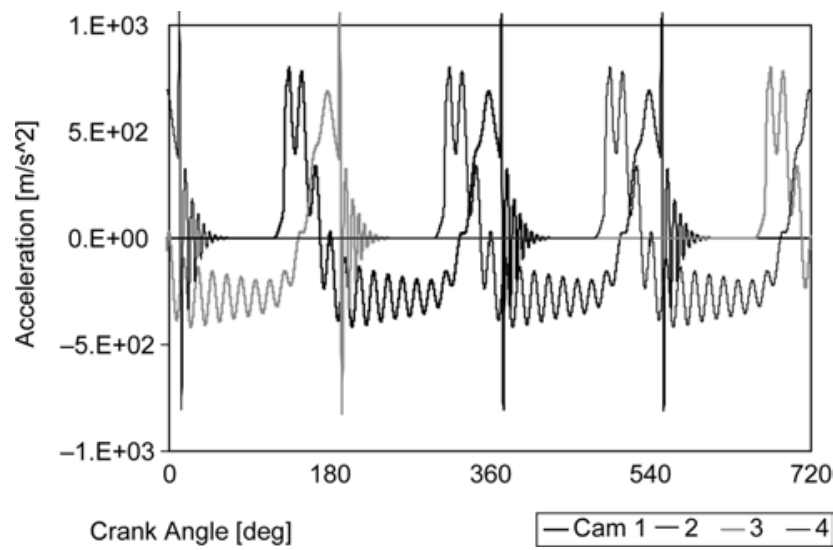

Fig. 16 Tappet acceleration for $200 \mathrm{r} / \mathrm{min}$

lower influence upon the dynamic response of the valvetrain than would initially be expected. The explanation is that at the end of the cam event the contact between the cam and the tappet is lost owing to the cam-tappet clearance. However, the current polynomial valve lift model does not include a model for the cam ramp. The connection between the cam and the tappet is lost instantly in the mathematical model, and by a gradually increasing gap in the real engine. Consequently, in the experimental engine, owing to a very high level of vibration, while the cam-tappet clearance is still very small, the end of the event is followed by a series of successive impacts between the cam and the tappet.

In Fig. 17(a) the centre-line of the undeformed camshaft is represented by the abscissa in the figure at a value of zero deformation. Since, at any instant of time, at least one cam is in operational cycle, the camshaft is always subjected to some amount of deformation. This is shown in the figure by the off-centre location of the centre of the cam from the undeformed

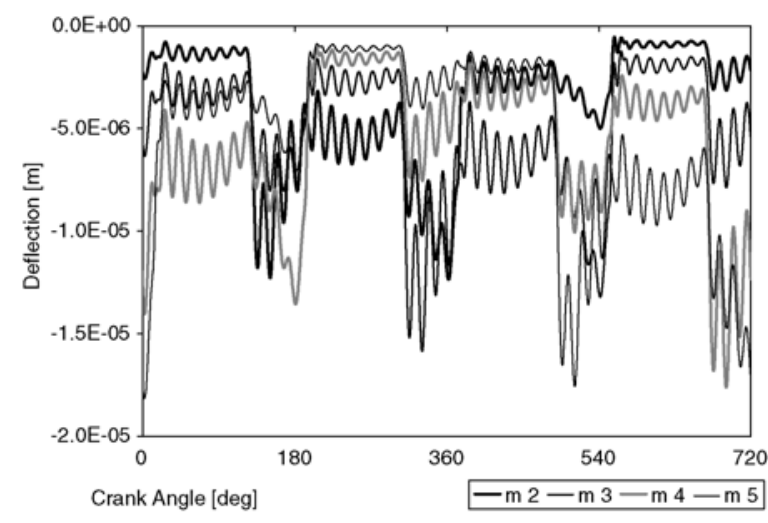

(a) bending

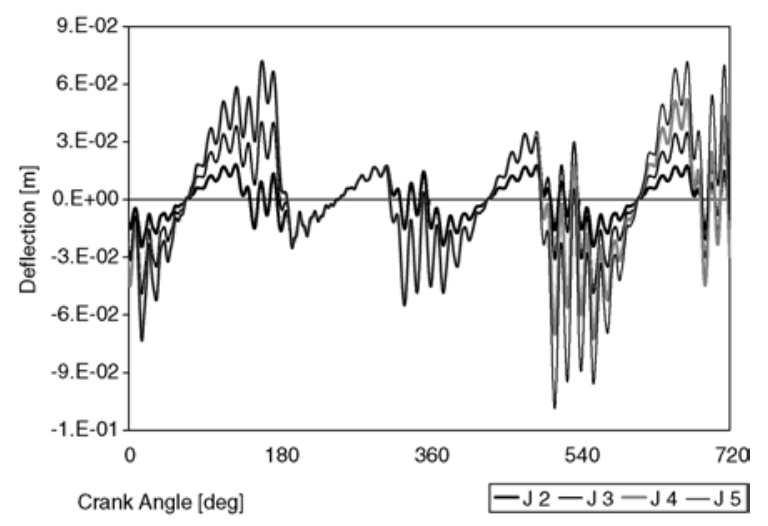

(b) torsional

Fig. 17 Camshaft deflection at cam positions at $2000 \mathrm{r} / \mathrm{min}$ 

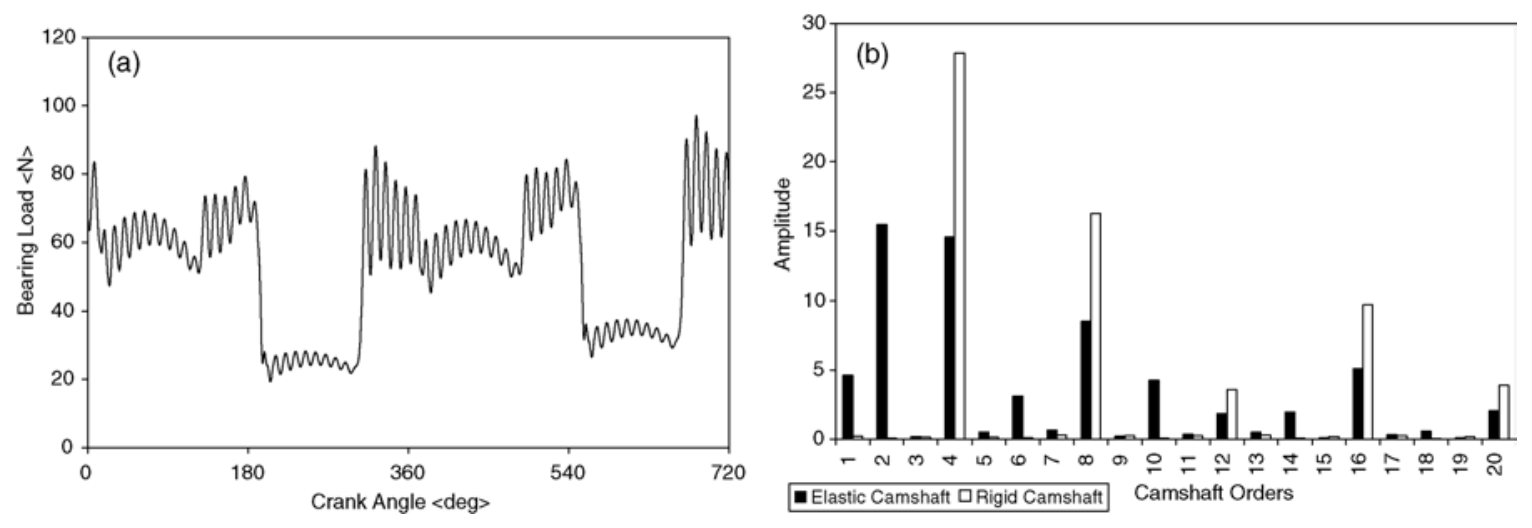

Fig. 18 Bearing load at $1400 \mathrm{r} / \mathrm{min}$

location. This displacement is, however, for most of the cam event, smaller than the camshaft bearing clearance and does not result in bearing shell deformation. However, the much higher amplitude of the deflection occurring during the cam operation is the one that results in camshaft bearing shell deformation, and consequently additional calculations should be performed to include this deflection in a bearing lubrication model. Since each bearing brings into the dynamic system additional stiffness, the influence of each cam is felt mainly by its neighbours and less so at any distant location on the camshaft.

Figure 17(b) shows the predicted torsional vibrations along the camshaft. Since the model assumes a constant angular velocity for the cam gear, the angular deflection of the first bearing is the smallest, and the deflection progressively increases for the following bearings.

Figure 18(a) shows the force applied on the third camshaft bearing during valvetrain operation. An effective way of fully appreciating the implications of using an elastic camshaft in the valvetrain dynamic model is to compare the predicted bearing behaviour for the elastic and rigid camshaft models. For the rigid camshaft equipped with rigid bearings, all the bearing reactions are equal, their magnitude is equal with a fifth of the force applied on the camshaft (see Fig. 12), and they oppose the cam-tappet contact force. On the other hand, for the case of an elastic camshaft, each bearing behaves differently to the others. For this analysis, the middle bearing has been chosen as a representative bearing.

Figure 18(b) shows the spectra of the predicted response for the third camshaft bearing in the case of an elastic camshaft, together with the response predicted for the equivalent rigid camshaft. In the simplified case of the rigid camshaft (shown in grey in Fig. 18(b)), only the major harmonic orders $(4,8,12, \ldots)$ are obtained, and the small values predicted for the rest of the harmonic orders are due to the approximate nature of the Fourier decomposition. However, for the case of an elastic camshaft (shown in black in Fig. 18(b)), it is important to note that, while the contributions at the even orders are diminished, all the intermediate orders are also well represented (odd camshaft orders, i.e. half engine orders, referred to as engine roughness).

The reduction in even engine orders is due to the reduced inertial imbalance with elastic camshafts of lighter construction, a driving force behind modern engine development philosophy. The engine roughness contribution is the result of the use of materials of lower elastic moduli (i.e. an elastodynamic problem, leading to noise and vibration issues just becoming appreciated in industry). The analysis in this paper shows that an 'optimum' has to be found in the interplay between inertial imbalance and the elasticity of system for any given engine power output and package space.

\section{ACKNOWLEDGEMENTS}

The authors would like to acknowledge the technical and financial support of the US Army National Automotive Center, TARDEC, Warren, Michigan, and the SKF Engineering Research Centre in Nieuwegein, the Netherlands.

\section{REFERENCES}

1 Teodorescu, M. Modular approach for valve-train dynamics and friction simulation with in situ experimental validation. PhD thesis, Wayne State University, Detroit, Michigan, 2003.

2 Teodorescu, M. and Tarazam D. Numerical prediction and experimental investigation of cam-flat tappet lubricated contact with tappet spin. Proc. Instn Mech. Engrs, Part K: J. Multi-body Dynamics, 2004, in, press. 
3 Koster, M. P. Effect of flexibility of driving shaft on the dynamic behaviour of a Cam mechanism. Trans. ASME J. Engng for Industry, 1975, 595-602.

4 Kushwaha, M., Rahnejat, H., and Jin, Z. M. Valve-train dynamics: a simplified tribo-elasto-multi-body analysis. Proc. Instn Mech. Engrs, Part K: J. Multi-body Dynamics, 2000, 214(K2), 95-110.

5 Dowson, D., Taylor, C. M., and Zhu, G. A transient elastohydradynamic lubrication analysis of a cam and follower. J. Phys. D: Appl. Phys., 1992, 25, 313-320.

6 Kushwaha, M. and Rahnejat, H. Transient elastohydrodynamic lubrication of finite line conjunction of cam to follower concentrated contact. J. Phys. D: Appl. Phys., 2002, 35(3), 2872-2890.

7 Mostofi, A. and Gohar, R. Oil film thickness and pressure distribution in elastohydrodynamic point contact. J. Mech. Engng Sci., 1982, 24(4), 173-182.

8 Rahnejat, H. Influence of Vibration on the Oil Film in Concentrated Contacts, 1984, Imperial College of Science and Technology, London).

9 Mehdigoli, H., Rahnejat, H., and Gohar, R. Vibration response of wavy surfaced disc in elasto-hydrodynamic rolling contact. Wear, 1990, 139, 1-15.

10 Dareing and Johnson. Fluid film damping of rolling contact vibrations. J. Mech. Engng Sci. 1975, 17(4), 214-218.

11 Greenwood, J. A. and Tripp, J. H. The contact of two nominally flat rough surfaces. Proc. Instn Mech. Engrs, 1970-71, 185(48/71), 625-633.

12 Teodorescu, M. et al. Simplified elasto-hydrodynamic friction model of the cam-tappet contact. SAE technical paper 2003-01-0985, 2003.

13 Evans, C. R. and Johnson, K. L. The rheological properties of elastohydrodynamic lubricants. Proc. Instn Mech. Engrs. Part C: J. Mechanical Engineering Science, 1986, 200(C5), 303-312.

14 Teodorescu, M. et al. Experimental analysis of dynamics and friction in valve-train systems. SAE technical paper 2002-01-0484, 2002.

15 Chen, J.-S. Dynamic analysis of a 3D finger follower valvetrain system coupled with flexible camshafts. SAE technical paper 2000-01-0909, 2000.

16 Rahnejat, H. Multi-body Dynamics: Vehicles, Machines and Mechanisms, July 1998 (Professional Engineering Publications, London, and Society of Automotive Engineers, Warrendale, Pennsylvania).

17 Kushwaha, M., Rahnejat, H., and Gohar, R. Aligned and misaligned contacts of rollers to races in elastohydrodynamic finite line conjunctions. Proc. Instn Mech. Engrs, Part C: J. Mechanical Engineering Science, 2002, 216(11), 1051-1070.

\section{APPENDIX 1}

\section{Notation}

A Hertzian contact area $\left(\mathrm{m}^{2}\right)$

$A_{\mathrm{a}} \quad$ actual asperity contact area $\left(\mathrm{m}^{2}\right)$

$c_{\mathrm{e} i} \quad$ equivalent damping coefficient $(\mathrm{N} \mathrm{s} / \mathrm{m})$ $c_{i} \quad$ damping coefficient ( $\left.\mathrm{N} \mathrm{s} / \mathrm{m}\right)$

$E_{1}, E_{2} \quad$ elastic moduli for cam and follower materials $(\mathrm{Pa})$

$E^{\prime} \quad \frac{1}{E^{\prime}}=\frac{1}{2}\left\{\frac{1-v^{2}}{E_{1}}+\frac{1-v^{2}}{E_{2}}\right\}$

$f_{i}$

$F_{\mathrm{b}}$

$F_{\mathrm{f}}$

$F_{n}(\lambda)$

friction forces associated with mass $i(\mathrm{~N})$

boundary friction force $(\mathrm{N})$

friction force between the cam and

tappet $(\mathrm{N})$

statistical functions

$=\frac{1}{\sqrt{2 \pi}} \int_{\gamma}^{\infty}(s-\lambda)^{n} \mathrm{e}^{-s^{2} / 2} \mathrm{~d} s(n=2$ or $5 / 2)$

$F_{\mathrm{v}} \quad$ viscous friction force (N)

$G^{*} \quad$ non-dimensional material parameter $=\alpha E^{\prime}$

$h_{0} \quad$ central oil film thickness (m)

$i$

$I_{R}$

$k$

$k_{\mathrm{e} i}$

$k_{i}$

K.E.

$L$

order number or compliance identity

rocker arm moment of inertia $\left(\mathrm{kg} \mathrm{m}^{2}\right)$

contact stiffness $(\mathrm{N} / \mathrm{m})$

equivalent stiffness $(\mathrm{N} / \mathrm{m})$

stiffness $(\mathrm{N} / \mathrm{m})$

rocker arm kinetic energy $(\mathrm{J})$

cam width (m)

$m \quad$ pressure coefficient of the boundary shear strength

$m_{1} \quad$ valve assembly equivalent mass (kg)

$m_{2} \quad$ pushrod and tappet equivalent mass (kg)

$r$

the distance between rocker arm bearing

and rocker arm-pushrod contact (m)

$R_{\mathrm{b}} \quad$ base circle radius (m)

$R_{\mathrm{c}} \quad$ instantaneous curvature radius (m)

$s \quad$ floor movement (tappet lift) (m)

$u$

$u_{1}$

$u_{2}$

$U^{*}$

$w_{\mathrm{s}}^{*}$

$W$

$W_{\mathrm{a}}$

$W^{*}$

$W$

$X, Y$

$y$

$z$

speed of entraining motion $(\mathrm{m} / \mathrm{s})$

instantaneous cam surface speed $(\mathrm{m} / \mathrm{s})$

surface speed of the flat follower $(\mathrm{m} / \mathrm{s})$

non-dimensional speed $=\left(\eta_{\mathrm{O}} u_{\mathrm{e}}\right) /\left(E^{\prime} R_{\mathrm{c}}\right)$

squeeze-roll ratio

contact load $(\mathrm{N})$

load carried by asperities in the contact area $(\mathrm{N})$

non-dimensional load $=W /\left(E^{\prime} R_{\mathrm{c}} L\right)$

oil-film pressure $\left(\mathrm{N} / \mathrm{m}^{2}\right)$

global frame of reference

mass $m_{1}$ movement (m)

mass $m_{2}$ movement (m)

piezoviscosity index $\left(\mathrm{m}^{2} / \mathrm{N}\right)$

$\alpha$

$\beta$

$\gamma, \gamma$

asperity radius of curvature $(\mathrm{m})$

rate of change in shear stress with pressure and the phase vector of cylinder firing in the camstar contact deflection (m)

time increment (s)

surface density of asperity peaks $\left(1 / \mathrm{m}^{2}\right)$ atmospheric dynamic viscosity of the oil (Pa s)

film thickness parameter $=h_{0} / \sigma$ 
Poisson's ratio

combined surface roughness (m) rocker arm oscillation angle (rad) shear strength $(\mathrm{Pa})$

limiting shear stress $(\mathrm{Pa})$

Eyring stress of the oil (Pa)

camshaft angular velocity $(\mathrm{rad} / \mathrm{s})$

\section{APPENDIX 2}

\section{Rocker arm equivalent mass}

During valve train operation, the rocker arm is oscillating around a fixed bearing and the kinetic energy can be written as:

$$
K . E .=\frac{1}{2} I_{R} \theta_{R}
$$

The equivalent mass translates together with the pushrod and the relation between the rocker arm angle and pushrod lift is: $y=r \sin \theta_{R}$. Consequently the kinetic energy can be written as:

$$
K . E .=\frac{1}{2} I_{R} \cdot \frac{\dot{y}^{2}}{r^{2} \cos ^{2} \theta_{R}}
$$

The rocker arm oscillation angle is smaller than $12^{\circ}$ [...] and the error introduced by considering $\cos ^{2} \theta \approx 1$ is smaller than $4 \%$.

$$
\text { K.E. }=\frac{1}{2} I_{R} \cdot \frac{\dot{y}^{2}}{r^{2}}=\frac{1}{2} m_{R} \dot{y}^{2} \rightarrow m_{R}=\frac{I_{R}}{r^{2}}
$$

The current work considered the rocker arm equivalent mass equally distributed between the two concentrated masses of the dynamic system: $m_{1}$ and $m_{2}$. 